\title{
Heavy Quark Potential with Hyperscaling Violation
}

\author{
Zi-qiang Zhang, ${ }^{1}$ Chong $\mathrm{Ma},{ }^{1}$ De-fu Hou, ${ }^{2}$ and Gang Chen ${ }^{1}$ \\ ${ }^{1}$ School of Mathematics and Physics, China University of Geosciences, Wuhan 430074, China \\ ${ }^{2}$ Institute of Particle Physics and Key Laboratory of Quark and Lepton Physics (MOE), Central China Normal University, \\ Wuhan 430079, China \\ Correspondence should be addressed to Zi-qiang Zhang; zhangzq@cug.edu.cn
}

Received 19 December 2016; Accepted 23 January 2017; Published 6 March 2017

Academic Editor: Song He

Copyright (c) $2017 \mathrm{Zi}$-qiang Zhang et al. This is an open access article distributed under the Creative Commons Attribution License, which permits unrestricted use, distribution, and reproduction in any medium, provided the original work is properly cited. The publication of this article was funded by SCOAP $^{3}$.

\begin{abstract}
We investigate the behavior of the heavy quark potential in the backgrounds with hyperscaling violation. The metrics are covariant under a generalized Lifshitz scaling symmetry with the dynamical Lifshitz parameter $z$ and hyperscaling violation exponent $\theta$. We calculate the potential for a certain range of $z$ and $\theta$ and discuss how it changes in the presence of the two parameters. Moreover, we add a constant electric field to the backgrounds and study its effects on the potential. It is shown that the heavy quark potential depends on the nonrelativistic parameters. Also, the presence of the constant electric field tends to increase the potential.
\end{abstract}

\section{Introduction}

AdS/CFT [1-3], which relates a $d$-dimensional quantum field theory with its dual gravitational theory, living in $(d+1)$ dimensions, has yielded many important insights into the dynamics of strongly coupled gauge theories. For reviews, see [4-15] and references therein.

Due to the broad application of this characteristic, many authors have considered the generalizations of the metrics dual to field theories. One of such generalizations is to use metric with hyperscaling violation. Usually, the metric is considered to be an extension of the Lifshitz metric and has a generic Lorentz violating form [16-20]. As we know, Lorentz symmetry represents a foundation of both general relativity and the standard model, so one may expect new physics from Lorentz invariance violation. For that reason, the metrics with hyperscaling violation have been used to describe the string theory [21-25] and holographic superconductors [26-29] as well as QCD [30-32].

The heavy quark potential of QCD is an important quantity that can probe the confinement mechanism in the hadronic phase and the meson melting in the plasma phase. In addition, it has been measured in great detail in lattice simulations. The heavy quark potential for $\mathcal{N}=4$ SYM theory was first obtained by Maldacena in his seminal work [33]. Interestingly, it is shown that for the $A d S_{5}$ space the energy shows a purely Coulombian behavior which agrees with a conformal gauge theory. This proposal has attracted lots of interest. After [33], there are many attempts to address the heavy quark potential from the holography. For example, the potential at finite temperature has been studied in $[34,35]$. The subleading order correction to this quantity is discussed in $[36,37]$. The potential has also been investigated in some AdS/QCD models [38, 39]. Other important results can be found, for example, in [40-44].

Although the theories with hyperscaling violation are intrinsically nonrelativistic, we can use them as toy models for quarks from the holography point of view. In addition, one can expect that the results obtained from these theories provide qualitative insights into analogous questions in QCD. In this paper, we will investigate the heavy quark potential in the Lifshitz backgrounds with hyperscaling violation. We want to know what will happen to the potential if we have the quark-antiquark pair in such backgrounds? More specifically, we would like to see how the potential changes in the presence of the nonrelativistic parameters. In addition, we will add a 
constant electric field to the backgrounds and study how it affects the potential. These are the main motivations of the present work.

We organize the paper as follows. In the next section, the backgrounds of the hyperscaling violation theories in [25] are briefly reviewed. In Section 3, we study the heavy quark potential in these backgrounds in terms of the $z$ and $\theta$ parameters. In Section 4, we investigate a constant electric field effect on the heavy quark potential. The last part is devoted to conclusion and discussion.

\section{Hyperscaling Violation Theories}

Let us begin with a brief review of the background in [25]. It has been argued that the Lorentz invariance is broken in this background metric. Although charge densities induce a trivial (gapped) behavior at low energy/temperature, there still exist special cases where there are nontrivial IR fixed points (quantum critical points) where the theory is scale invariant. Usually, the metric is expressed as [21]

$$
d s^{2}=u^{\theta}\left[-\frac{d t^{2}}{u^{2 z}}+\frac{b_{0} d u^{2}+d x^{i} d x^{i}}{u^{2}}\right],
$$

where $b_{0}=\ell^{2}$ with $\ell$ being the IR scale. The above metric is covariant under a generalized Lifshitz scaling symmetry; that is,

$$
\begin{gathered}
t \longrightarrow \lambda^{z} t, \\
u \longrightarrow \lambda u, \\
x^{i} \longrightarrow \lambda x^{i}, \\
d s^{2} \longrightarrow \lambda^{-\theta} d s^{2},
\end{gathered}
$$

where $z$ is called the dynamical Lifshitz parameter or the dynamical critical exponent which characterizes the behavior of system near the phase transition. $\theta$ stands for the hyperscaling violation exponent which is responsible for the nonstand scaling of physical quantities and controls the transformation of the metric. The scalar curvature of these geometries is

$$
R=-\frac{3 \theta^{2}-4(z+3) \theta+2\left(z^{2}+3 z+6\right)}{b_{0}} u^{-\theta}
$$

The geometries are flat when $\theta=2$ and $z=0,1$. The geometry is Ricci flat when $\theta=4$ and $z=3$. The geometry is in Rindler coordinates when $\theta=0$ and $z=1$. Usually, the above special solutions violate the Gubser bound conditions [25]. In addition, the pure Lifshitz case is related to $\theta=0$.

By using a radical redefinition

$$
u=(2-z) r^{1 /(2-z)}
$$

and rescaling $t$ and $x^{i}$, we have the following metric:

$$
\begin{aligned}
& d s^{2} \sim r^{(\theta-2) /(2-z)}\left[-f(r) d t^{2}+\frac{d r^{2}}{f(r)}+d x^{i} d x^{i}\right], \\
& f(r)=f_{0} r^{2(1-z) /(2-z)}, \\
& \text { with } f_{0}=(2-z)^{2(1-z)} \text {. }
\end{aligned}
$$

In the presence of hyperscaling violations, the energy scale is

$$
E \simeq u^{(\theta-2 z) / 2} \simeq r^{(\theta-2 z) / 2(2-z)} .
$$

For the generalized scaling solutions of (5), the Gubser bound conditions are as follows:

$$
\begin{aligned}
\frac{2 z+3(2-\theta)}{2(z-1)-\theta} & >0, \\
\frac{z-1}{2(z-1)-\theta}>0, & >0 .
\end{aligned}
$$

Also, to consider the thermodynamic stability, one needs

$$
\frac{z}{2(z-1)-\theta}>0
$$

More discussions about other generalized Lifshitz geometries can be found in [25].

The generalizations of (5) to include finite temperature can be written as

$$
\begin{aligned}
d s^{2} & \sim\left(\frac{r}{\ell}\right)^{-\alpha}\left[-f(r) d t^{2}+\frac{d r^{2}}{f(r)}+d x^{i} d x^{i}\right], \\
f(r) & =f_{0}\left(\frac{r}{\ell}\right)^{2 \beta} h, \\
h & =1-\left(\frac{r}{r_{h}}\right)^{\gamma},
\end{aligned}
$$

where $\alpha=(\theta-2) /(z-2), \beta=(z-1) /(z-2)$, and $\gamma=$ $(2 z+3(2-\theta)) / 2(2-z)$.

The Hawking temperature is

$$
T=\frac{f_{0}}{8 \pi \ell}\left(\frac{r_{h}}{\ell}\right)^{z /(z-2)}\left|\frac{2 z-3 \theta+6}{z-2}\right| .
$$

\section{Heavy Quark Potential}

In the holographic description, the heavy quark potential is given by the expectation value of the static Wilson loop

$$
W(C)=\frac{1}{N} \operatorname{Tr} P e^{i \int A_{\mu} d x^{\mu}},
$$

where $C$ is a closed loop in a 4-dimensional space time and the trace is over the fundamental representation of the $\mathrm{SU}(N)$ group. $A_{\mu}$ is the gauge potential and $P$ enforces the path ordering along the loop $C$. The heavy quark potential can be extracted from the expectation value of this rectangular Wilson loop in the limit $\mathscr{T} \rightarrow \infty$ :

$$
\langle W(C)\rangle \sim e^{-\mathscr{T V}} .
$$

On the other hand, the expectation value of Wilson loop in (12) is given by

$$
\langle W(C)\rangle \sim e^{-S_{c}},
$$


where $S_{c}$ is the regularized action. Therefore, the heavy quark potential can be expressed as

$$
V=\frac{S_{c}}{\mathscr{T}}
$$

We now analyze the heavy quark potential using the metric of (9). The string action can reduce to the NambuGoto action:

$$
S=-\frac{1}{2 \pi \alpha^{\prime}} \int d \tau d \sigma \sqrt{-\operatorname{det} g_{\alpha \beta}}
$$

where $g$ is the determinant of the induced metric on the string world sheet embedded in the target space; that is,

$$
g_{\alpha \beta}=G_{\mu \nu} \frac{\partial X^{\mu}}{\partial \sigma^{\alpha}} \frac{\partial X^{\nu}}{\partial \sigma^{\beta}}
$$

where $X^{\mu}$ and $G_{\mu \nu}$ are the target space coordinates and the metric, and $\sigma^{\alpha}$ with $\alpha=0,1$ parameterize the world sheet.

Using the parametrization $X^{\mu}=(t, x, 0,0, r), \sigma=x$, $\tau=t$ and $r=r(x)$, we extremize the open string worldsheet attached to a static quark at $x=+L / 2$ and an antiquark at $x=-L / 2$. Then the induced metric of the fundamental string is given by

$$
g_{\alpha \beta}=b(r)\left(\begin{array}{cc}
-f(r) & 0 \\
0 & 1+\frac{\dot{r}^{2}}{f(r)}
\end{array}\right),
$$

with $b(r)=(r / \ell)^{-\alpha}, \dot{r}=d r / d x$.

Plugging (17) into (15), the Euclidean version of NambuGoto action in (9) becomes

$$
S=\frac{\mathscr{T}}{2 \pi \alpha^{\prime}} \int d x \sqrt{b^{2}(r)\left[f(r)+\dot{r}^{2}\right]} .
$$

We now identify the Lagrangian as

$$
\mathscr{L}=\sqrt{b^{2}(r)\left[f(r)+\dot{r}^{2}\right]} .
$$

Note that $\mathscr{L}$ does not depend on $x$ explicitly, so the corresponding Hamiltonian will be a constant of motion; that is,

$$
H=\frac{\partial \mathscr{L}}{\partial \dot{r}} \dot{r}-\mathscr{L}=\text { Constant }=C .
$$

This constant can be found at special point $r(0)=r_{c}$, where $r_{c}^{\prime}=0$, as

$$
H=-\sqrt{f\left(r_{c}\right) b^{2}\left(r_{c}\right)}
$$

and then a differential equation is derived:

$$
\dot{r}=\sqrt{\frac{f^{2}(r) b^{2}(r)-f(r) f\left(r_{c}\right) b^{2}\left(r_{c}\right)}{f\left(r_{c}\right) b^{2}\left(r_{c}\right)}},
$$

with

$$
\begin{aligned}
f\left(r_{c}\right) & =f_{0}\left(\frac{r_{c}}{\ell}\right)^{2 \beta} h_{1}, \\
h_{1} & =1-\left(\frac{r_{c}}{r_{h}}\right)^{\gamma}, \\
b\left(r_{c}\right) & =\left(\frac{r_{c}}{\ell}\right)^{-\alpha} .
\end{aligned}
$$

By integrating (22) the separation length $L(\theta, z)$ of quarkantiquark pair becomes

$L(\theta, z)$

$$
=2 \int_{0}^{r_{c}} d r \sqrt{\frac{f\left(r_{c}\right) b^{2}\left(r_{c}\right)}{f^{2}(r) b^{2}(r)-f(r) f\left(r_{c}\right) b^{2}\left(r_{c}\right)}} .
$$

On the other hand, plugging (22) into the Nambu-Goto action of (18), one finds the action of the heavy quark pair:

$$
S=\frac{\mathscr{T}}{\pi \alpha^{\prime}} \int_{0}^{r_{c}} d r b^{2}(r) \sqrt{\frac{f(r)}{f(r) b^{2}(r)-f\left(r_{c}\right) b^{2}\left(r_{c}\right)}}
$$

This action is divergent, but the divergences can be avoided by subtracting the inertial mass of two free quarks, given by

$$
S_{0}=\frac{\mathscr{T}}{\pi \alpha^{\prime}} \int_{0}^{r_{h}} d r b(r)
$$

Subtracting this self-energy, the regularized action is obtained:

$$
S_{c}=S-S_{0}
$$

Applying (14), we end up with the heavy quark potential with hyperscaling violation:

$$
\begin{aligned}
& V(\theta, z)=\frac{1}{\pi \alpha^{\prime}} \int_{0}^{r_{c}} d r \\
& \cdot\left[b^{2}(r) \sqrt{\frac{f(r)}{f(r) b^{2}(r)-f\left(r_{c}\right) b^{2}\left(r_{c}\right)}}-b(r)\right] \\
& \quad-\frac{1}{\pi \alpha^{\prime}} \int_{r_{c}}^{r_{h}} d r b(r) .
\end{aligned}
$$

Note that the potential $V(z)$ in the Lifshitz space-time [ 45 , 46] can be derived from (28) if we neglect the effect of the hyperscaling violation exponent by plugging $\theta=0$ in (28). Also, in the limit $(\theta=0, z=1),(28)$ can reduce to the finite temperature case in $[34,35]$.

Before evaluating the heavy quark potential of (28), we should pause here to determine the allowed region for $z$ and $\theta$ at hand. The space boundary is considered at $r=0$, consequently $\alpha>0$. To avoid (28) being ill-defined, it is required that $\gamma>0$. Moreover, one should consider the 


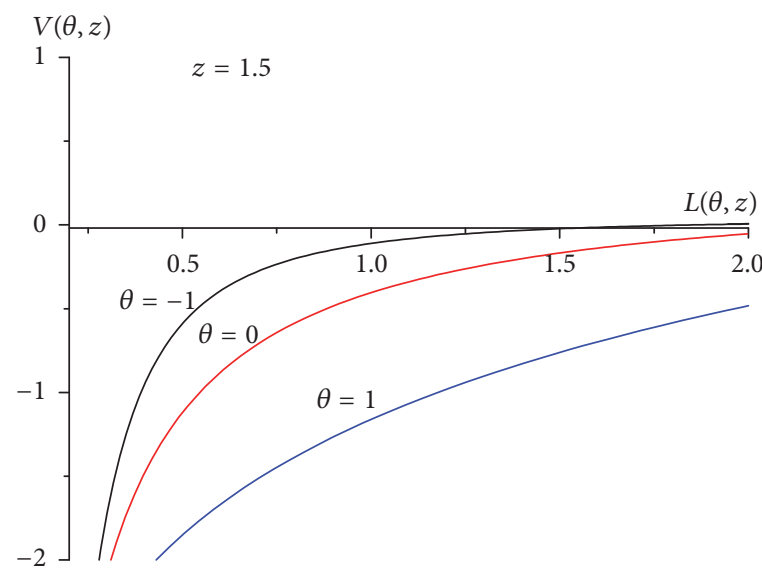

(a)

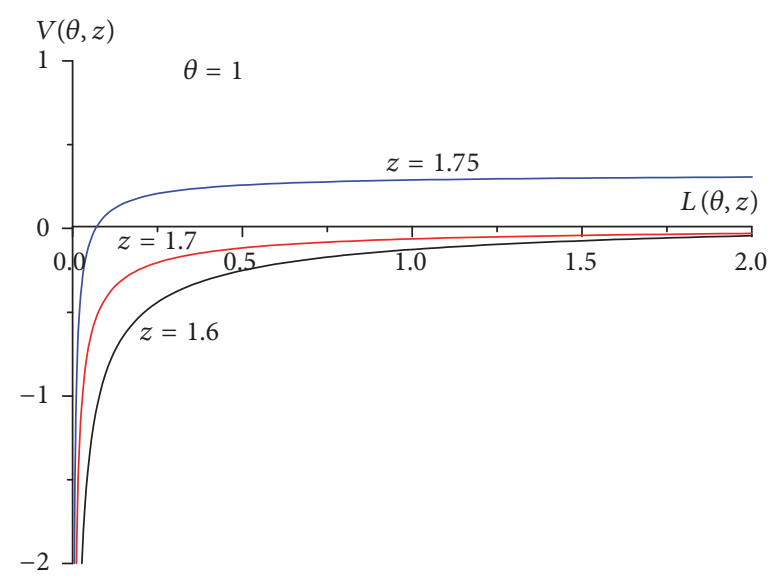

(b)

Figure 1: Plots of $V(\theta, z)$ versus $L(\theta, z)$. (a) $z=1.5$; from top to bottom $\theta=-1,0,1$, respectively; (b) $\theta=1$; from top to bottom $z=$ $1.75,1.7,1.6$, respectively.

Gubser conditions of (7) and the thermodynamic stability condition of (8). With these restrictions, one finds

$$
\begin{aligned}
& 1<z<2, \\
& \theta<2, \\
& \theta<z,
\end{aligned}
$$

and then one can choose the values of $z$ and $\theta$ in such a range.

In Figure 1, we plot the potential $V(\theta, z)$ versus distance $L(\theta, z)$ with different $z, \theta$. In Figure 1(a), the dynamical exponent is $z=1.5$ and from top to bottom the hyperscaling violation exponent is $\theta=-1,0,1$, respectively. In Figure 1(b), $\theta=1$ and from top to bottom $z=1.75,1.7,1.6$, respectively. From the figures, we can see clearly that by increasing $\theta$ the potential decreases. One finds also that increasing $z$ leads to increasing the potential. In other words, increasing $z$ and $\theta$ had different effects on the potential. Then one can change the potential by changing the values of these parameters. Therefore, the heavy quark potential depends on the nonrelativistic parameters.

To study how the potential changes with the temperature $T$, we show $V(\theta, z)$ as a function of $L(\theta, z)$ with $z=1.6$, $\theta=1$ in Figure 2. From (10), we can see that $T$ is a decreasing function of $z_{h}$ for $z=1.6$. So one finds in Figure 2 that increasing $T$ (or decreasing $z_{h}$ ) leads to increasing the potential. This result is consistent with the finding of $[34,35]$.

Moreover, to see the short distance behavior of the potential, we take the limit $L(\theta, z) T \rightarrow 0$ and find the following approximate formula:

$$
\begin{aligned}
L(\theta, z) & \simeq \frac{2 f_{0}^{-1 / 2}}{\alpha-2 \beta+1} r_{c}^{1-\beta}, \\
V(\theta, z) & \simeq \frac{\sqrt{\lambda}}{\pi(1-\alpha)} r_{c}^{1-\alpha},
\end{aligned}
$$

which yields

$$
V(\theta, z) \propto L(\theta, z)^{\theta-z}
$$

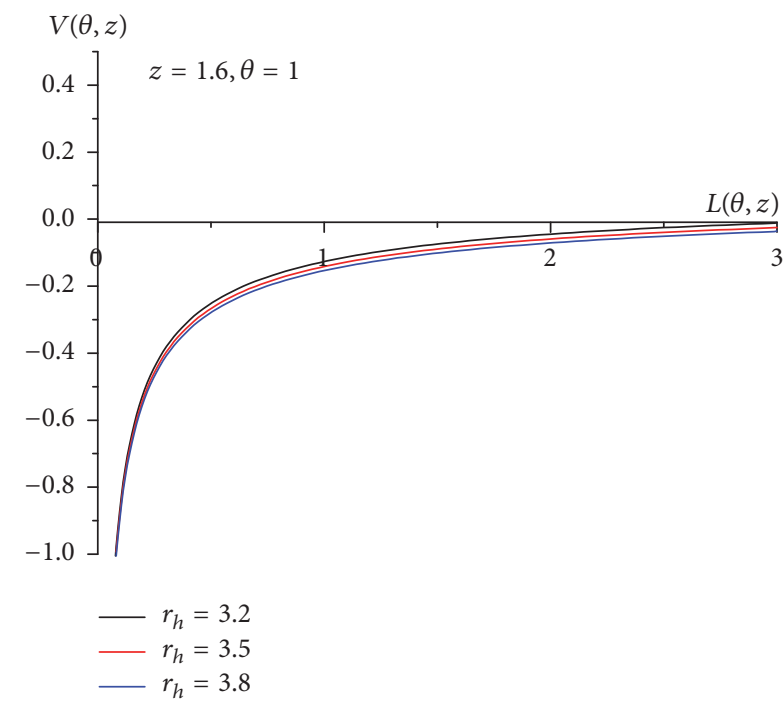

Figure 2: Plots of $V(\theta, z)$ versus $L(\theta, z)$ with $z=1.6, \theta=1$. From top to bottom $r_{h}=3.2,3.5,3.8$, respectively.

One can see that the potential is dependent on $z$ and $\theta$. In the special case of $\theta=0, z=1$ ( or $\alpha=2, \beta=0$ ), one finds that the potential is of Coulomb type:

$$
V(\theta, z) \simeq-\frac{\sqrt{\lambda}}{1.5 \pi L(\theta, z)},
$$

but for other cases, the potential may not be Coulombian.

\section{Effect of Constant Electric Field}

In this section, we study the effect of a constant electric field on the heavy quark potential following the method proposed in [12]. The constant $B$-field is along the $x^{1}$ and $x^{2}$ directions. As the field strength is involved in the equations of motion, this ansatz could be a good solution to supergravity as well as 
a simple way of studying the $B$-field correction. The constant $B$-field is added to the metric of (9) by the following form:

$$
B=B_{01} d t \wedge d x^{1}+B_{12} d x^{1} \wedge d x^{2},
$$

where $B_{01}$ and $B_{12}$ are assumed to be constants with $B_{01}=E$ being the NS-NS antisymmetric electric field and $B_{12}=H$ being the NS-NS antisymmetric magnetic field.

The constant $B$-field considered here is only turned on $x^{1}$ direction, which implies $H=0$. After adding an electric field to this background metric of (9), the string action is given by

$$
S=-\frac{1}{2 \pi \alpha^{\prime}} \int d \tau d \sigma \sqrt{-\operatorname{det}(g+b)_{\alpha \beta}}
$$

where $g_{\alpha \beta}$ is given in (17). $b_{\alpha \beta}=B_{\mu \nu} \partial_{\alpha} X^{\mu} \partial_{\beta} X^{\nu}$ is obtained as

$$
b_{\alpha \beta}=\left(\begin{array}{ll}
0 & 0 \\
0 & \xi
\end{array}\right) \text {, }
$$

and then the string action in (34) reads

$$
S=\frac{\mathscr{T}}{2 \pi \alpha^{\prime}} \int d x \sqrt{b^{2}(r) f(r)+b(r) f(r) \xi+b^{2}(r) \dot{r}^{2}} .
$$

Parallel to the case of the previous section, we have

$$
\dot{r}=\sqrt{\frac{A^{2}(r)-A(r) A\left(r_{c}\right)}{A\left(r_{c}\right) b^{2}(r)}},
$$

with

$$
\begin{gathered}
A(r)=b^{2}(r) f(r)+b(r) f(r) \xi, \\
A\left(r_{c}\right)=b^{2}\left(r_{c}\right) f\left(r_{c}\right)+b\left(r_{c}\right) f\left(r_{c}\right) \xi .
\end{gathered}
$$

We call again the separation length and the heavy quark potential as $L$ and $V$, respectively. One finds

$$
\begin{aligned}
L= & 2 \int_{0}^{r_{c}} d r \sqrt{\frac{A\left(r_{c}\right) b^{2}(r)}{A^{2}(r)-A(r) A\left(r_{c}\right)}} \\
V= & \frac{1}{\pi \alpha^{\prime}} \int_{0}^{r_{c}} d r\left[\sqrt{\frac{A(r) b^{2}(r)}{A(r)-A\left(r_{c}\right)}}-b(r)\right] \\
& -\frac{1}{\pi \alpha^{\prime}} \int_{r_{c}}^{r_{h}} d r b(r) .
\end{aligned}
$$

To see the effect of the constant electric field $\xi$ on the heavy quark potential in the backgrounds with hyperscaling violation, we plot the heavy quark potential as a function of the interdistance for $z=1.7, \theta=1$ with three different $\xi$ in Figure 3. In the plots from top to bottom $\xi=$ $3.0,1.5,0.1$, respectively. From the figures, we can see that the heavy quark potential increases with increasing $\xi$. In other words, the presence of the constant electric field leads to a smaller screening radius. This result can be understood by considering the relation between the potential and the viscosity of the medium. It was argued [47] that, increasing

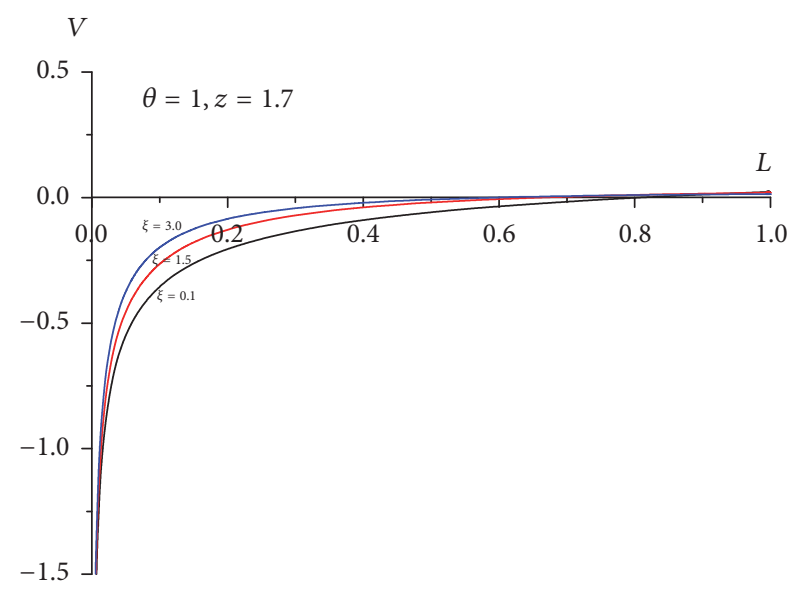

Figure 3: Plots of $V$ versus $L$ with different $\xi$. Here $z=1.7$ and $\theta=1$. From top to bottom $\xi=3.0,1.5,0.1$, respectively.

the viscosity, the screening of the potential due to the thermal medium weakens and so the potential decreases. On the other hand, the presence of the constant electric field tends to weaken the viscosity [12]. Thus, increasing the constant electric field leads to weakening the viscosity or increasing the potential.

\section{Conclusion and Discussion}

In this paper, we have investigated the heavy quark potential in the backgrounds with hyperscaling violation at finite temperature. These theories are strongly coupled with anisotropic scaling symmetry in the time and a spatial direction. Although the theories are not directly applicable to QCD, the features of them are similar to QCD. Therefore one can expect that the results obtained from these theories provide qualitative insights into analogous questions in QCD. In addition, an understanding of how the heavy quark potential changes by these theories may be useful for theoretical predictions.

In Section 3, we used the holographic description to calculate the heavy quark potential at finite temperature. We considered the space boundary at $r=0$ and discussed the potential for a certain range of $z$ and $\theta$ which satisfies the Gubser conditions and the thermodynamic stability condition. In is shown that increasing $z$ and $\theta$ had different effects: the potential increases as $z$ increases but it decreases as $\theta$ increases. As a result, the heavy quark potential depends on the nonrelativistic parameters. In Section 4, we added a constant electric field to the background metrics and study its effect on the heavy quark potential. We observed that the potential rises as the constant electric field increases. In other words, the presence of the constant electric field leads to increasing the heavy quark potential.

Finally, it is interesting to mention that the drag force [4] can also be studied in the backgrounds with hyperscaling violation. We will leave this for further study. 


\section{Competing Interests}

The authors declare that they have no competing interests.

\section{Acknowledgments}

This research is partly supported by the Ministry of Science and Technology of China (MSTC) under the 973 Project no. 2015CB856904(4). Zi-qiang Zhang and Gang Chen are supported by the NSFC under Grant no. 11475149. De-fu Hou is supported by the NSFC under Grant nos. 11375070 and 11521064.

\section{References}

[1] J. Maldacena, "The large-N limit of superconformal field theories and supergravity," International Journal of Theoretical Physics, vol. 38, no. 4, pp. 1113-1133, 1999.

[2] S. Gubser, I. Klebanov, and A. Polyakov, "Gauge theory correlators from non-critical string theory," Physics Letters B, vol. 428, no. 1-2, pp. 105-114, 1998.

[3] O. Aharony, S. S. Gubser, J. Maldacena, H. Ooguri, and Y. $\mathrm{Oz}$, "Large $N$ field theories, string theory and gravity," Physics Reports. A Review Section of Physics Letters, vol. 323, no. 3-4, pp. 183-386, 2000.

[4] C. P. Herzog, A. Karch, P. Kovtun, C. Kozcaz, and L. G. Yaffe, "Energy loss of a heavy quark moving through $N=4$ supersymmetric Yang-Mills plasma," Journal of High Energy Physics, vol. 2006, no. 7, 2006.

[5] C. P. Herzog, "Energy loss of a heavy quark from asymptotically AdS geometries," Journal of High Energy Physics, vol. 2006, no. 9, article no. 032, 2006.

[6] M. Ali-Akbari and U. Gürsoy, "Rotating strings and energy loss in non-conformal holography," Journal of High Energy Physics, vol. 2012, no. 1, 2012.

[7] S. S. Gubser, "Drag force in AdS/CFT", Physical Review. D. Third Series, vol. 74, 2006.

[8] E. Caceres and A. Guijosa, "Drag force in a charged $\mathcal{N}=4$ SYM plasma," Journal of High Energy Physics, vol. 2006, no. 11, article 077, 2006.

[9] J. Sadeghi and B. Pourhassan, "Drag force of moving quark at $\mathcal{N}=2$ supergravity," Journal of High Energy Physics, vol. 2008, no. 12 , article no. $026,2008$.

[10] K. B. Fadafan and H. Soltanpanahi, "Energy loss in a strongly coupled anisotropic plasma," Journal of High Energy Physics, vol. 2012, no. 10, article no. 085, 2012.

[11] M. Chernicoff, J. A. Garcia, and A. Guijosa, "The energy of a moving quark-antiquark pair in an $\mathcal{N}=4$ SYM plasma," Journal of High Energy Physics, vol. 2006, no. 9, article 068, 2006.

[12] T. Matsuo, D. Tomino, and W. Wen, "Drag force in SYM plasma with B field from AdS/CFT," Journal of High Energy Physics, vol. 2006, no. 10, article no. 055, 2006.

[13] R. Cai, S. Chakrabortty, S. He, and L. Li, "Some aspects of QGP phase in a hQCD model," Journal of High Energy Physics, vol. 2013, no. 2, article no. 068, 2013.

[14] M. Ali-Akbari and K. B. Fadafan, "Rotating mesons in the presence of higher derivative corrections from gauge-string duality," Nuclear Physics B, vol. 835, no. 1-2, pp. 221-237, 2010.

[15] U. Gürsoy and E. Kiritsis, "Exploring improved holographic theories for QCD: part I," Journal of High Energy Physics, vol. 2008, no. 2, article no. 032, 2008.
[16] T. Azeyanagi, W. Li, and T. Takayanagi, "On string theory duals of Lifshitz-like fixed points," Journal of High Energy Physics, vol. 2009, no. 6, article 084, 2009.

[17] K. Balasubramanian and K. Narayan, "Lifshitz spacetimes from AdS null and cosmological solutions," Journal of High Energy Physics, vol. 2010, no. 8, article 14, 2010.

[18] H. Singh, "Lifshitz/Schrödinger D p-branes and dynamical exponents," Journal of High Energy Physics, vol. 2012, no. 7, article 82, 2012.

[19] K. Narayan, "Lifshitz scaling and hyperscaling violation in string theory," Physical Review D, vol. 85, no. 10, Article ID 106006, 2012.

[20] P. Dey and S. Roy, "Intersecting D-branes and Lifshitz-like space-time," Physical Review D, vol. 86, 2012.

[21] B. Goutéraux and E. Kiritsis, "Generalized holographic quantum criticality at finite density," Journal of High Energy Physics, vol. 2011, no. 12, article no. 036, 2011.

[22] E. Perlmutter, "Domain wall holography for finite temperature scaling solutions," Journal of High Energy Physics, vol. 2011, no. 2, article 013, 2011.

[23] M. Alishahiha and H. Yavartanoo, "On holography with hyperscaling violation," Journal of High Energy Physics, vol. 2012, no. 11, 2012.

[24] J. Sadeghi, B. Pourhassan, and F. Pourasadollah, "Thermodynamics of Schrodinger black holes with hyperscaling violation," Physics Letters. B. Particle Physics, Nuclear Physics and Cosmology, vol. 720, no. 1-3, pp. 244-249, 2013.

[25] E. Kiritsis, "Lorentz violation, gravity, dissipation and holography," Journal of High Energy Physics, vol. 2013, no. 1, article 030, 2013.

[26] E. J. Brynjolfsson, U. H. Danielsson, L. Thorlacius, and T. Zingg, "Holographic superconductors with Lifshitz scaling," Journal of Physics. A. Mathematical and Theoretical, vol. 43, Article ID 065401, 2010.

[27] S. Sin, S. Xu, and Y. Zhou, "Holographic superconductor for a lifshitz fixed point," International Journal of Modern Physics A, vol. 26, no. 26, pp. 4617-4631, 2011.

[28] Y. Bu, "Holographic superconductors with $z=2$ Lifshitz scaling," Physical Review D, vol. 86, no. 4, Article ID 046007, 2012.

[29] Q. Pan and S.-J. Zhang, "Revisiting holographic superconductors with hyperscaling violation," The European Physical Journal C, vol. 76, article 126, 2016.

[30] J. Sadeghi and S. Heshmatian, "Jet quenching parameter with hyperscaling violation," The European Physical Journal C, vol. 74, no. 9, 2014.

[31] J. Sadeghi and S. Heshmatian, "Decay widths of large-spin mesons from the noncritical string/gauge duality," Physical Review D, vol. 84, no. 12, 2011.

[32] K. B. Fadafan and F. Saiedi, "Holographic Schwinger effect in non-relativistic backgrounds," The European Physical Journal C, vol. 75, no. 12, 2015.

[33] J. M. Maldacena, "Wilson loops in large $N$ field theories," Physical Review Letters, vol. 80, no. 22, pp. 4859-4862, 1998.

[34] A. Brandhuber, N. Itzhaki, J. Sonnenschein, and S. Yankielowicz, "Wilson loops in the large $N$ limit at finite temperature," Physics Letters B, vol. 434, no. 1-2, pp. 36-40, 1998.

[35] S. Rey, S. Theisen, and J. Yee, "Wilson-Polyakov loop at finite temperature in large- $\mathrm{N}$ gauge theory and anti-de Sitter supergravity," Nuclear Physics B, vol. 527, no. 1-2, pp. 171-186, 1998. 
[36] S. Chu, D. Hou, and H. Ren, “The subleading term of the strong coupling expansion of the heavy-quark potential in a $\mathcal{N}=4$ super Yang-Mills vacuum," Journal of High Energy Physics, vol. 2009, 2009.

[37] Z. Zhang, D. Hou, H. Ren, and L. Yin, "The subleading term of the strong coupling expansion of the heavy-quark potential in a $\mathcal{N}=4$ super Yang-Mills plasma," Journal of High Energy Physics, vol. 2011, no. 7, article 35, 2011.

[38] O. Andreev and V. I. Zakharov, "On heavy-quark free energies, entropies, Polyakov loop, and AdS/QCD," Journal of High Energy Physics, vol. 2007, no. 4, article no. 100, 2007.

[39] S. He, M. Huang, and Q. Yan, "Logarithmic correction in the deformed AdS5 model to produce the heavy quark potential and QCD beta function," Physical Review D, vol. 83, no. 4, Article ID 045034, 2011.

[40] J. Greensite and P. Olesen, "Remarks on the heavy quark potential in the supergravity approach," Journal of High Energy Physics, vol. 1998, 1998.

[41] F. Bigazzi, A. L. Cotrone, L. Martucci, and L. A. Pando Zayas, "Wilson loop, Regge trajectory, and hadron masses in a YangMills theory from semiclassical strings," Physical Review DParticles, Fields, Gravitation and Cosmology, vol. 71, no. 6, Article ID 066002, 2005.

[42] L. Martucci, "Semiclassical strings on confining backgrounds," Fortschritte der Physik. Progress of Physics, vol. 53, no. 7-8, pp. 936-942, 2005.

[43] Y. Kinar, E. Schreiber, and J. Sonnenschein, " $\overline{Q Q}$ potential from strings in curved space-time-classical results," Nuclear Physics. $B$, vol. 566, no. 1-2, pp. 103-125, 2000.

[44] S. He, M. Huang, and Q. Yan, "Heavy quark potential and QCD beta function from a deformed AdS5 model," Progress of Theoretical Physics Supplement, vol. 186, pp. 504-509, 2010.

[45] U. H. Danielsson and L. Thorlacius, "Black holes in asymptotically Lifshitz spacetime," Journal of High Energy Physics, vol. 2009, 2009.

[46] J. Klusoň, "Open string in a nonrelativistic background," Physical Review D, vol. 81, no. 10, Article ID 106006, 8 pages, 2010.

[47] J. Noronha and A. Dumitru, "Heavy quark potential as a function of shear viscosity at strong coupling," Physical Review $D$, vol. 80, Article ID 014007, 2009. 

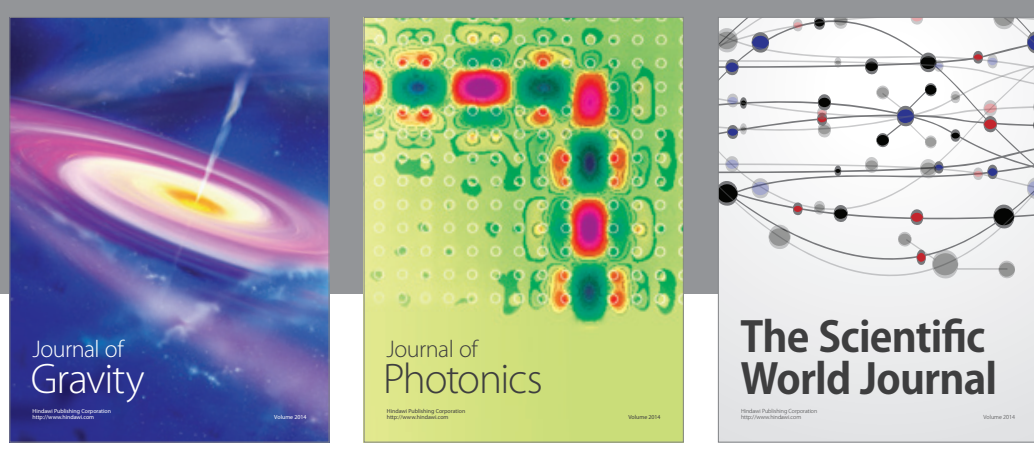

The Scientific World Journal
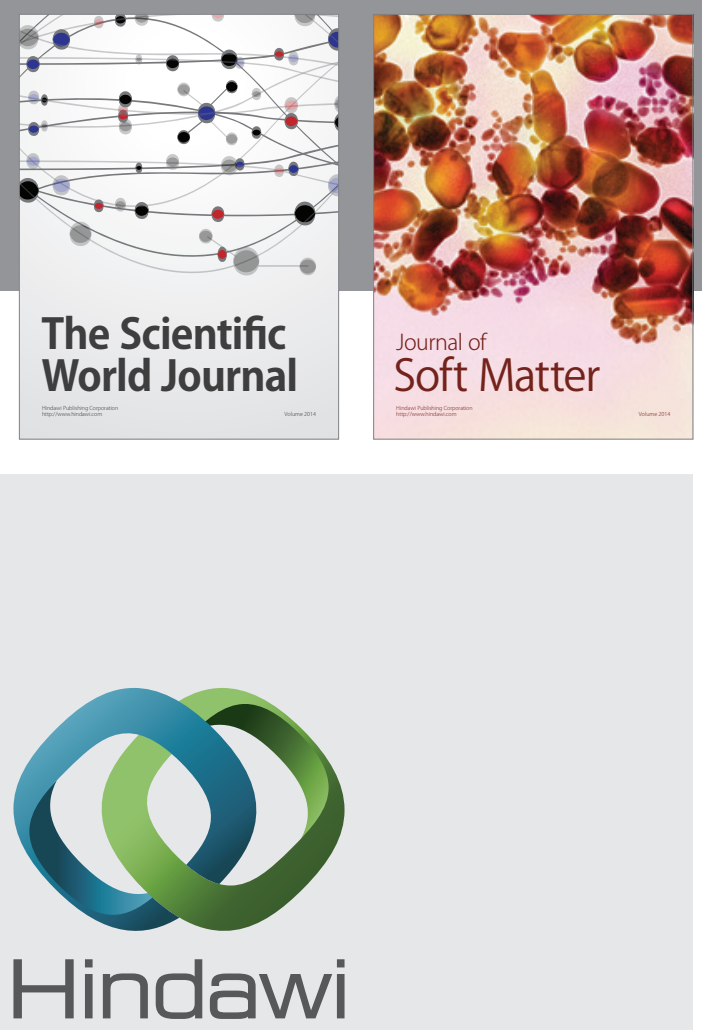

Submit your manuscripts at

https://www.hindawi.com
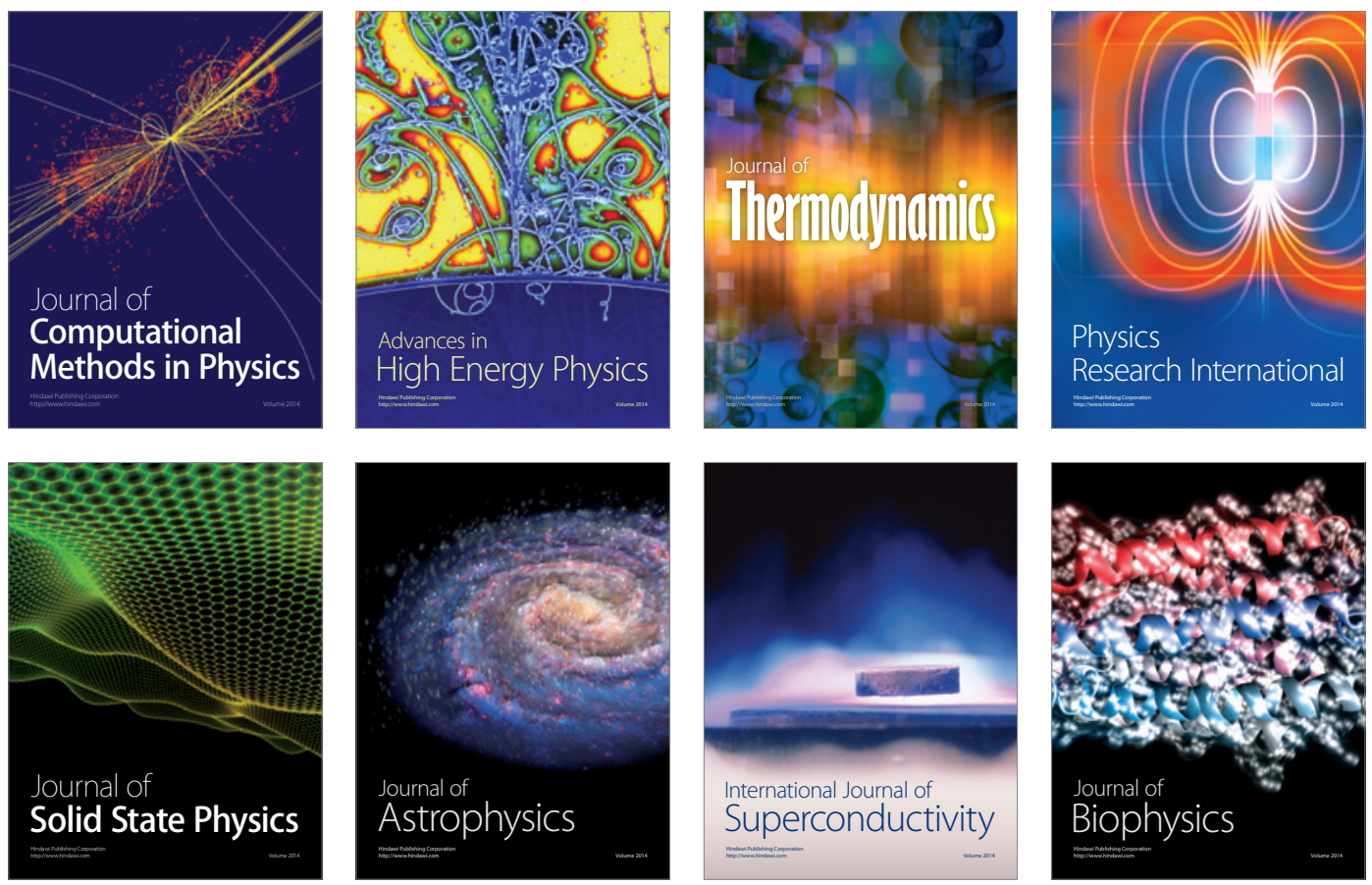
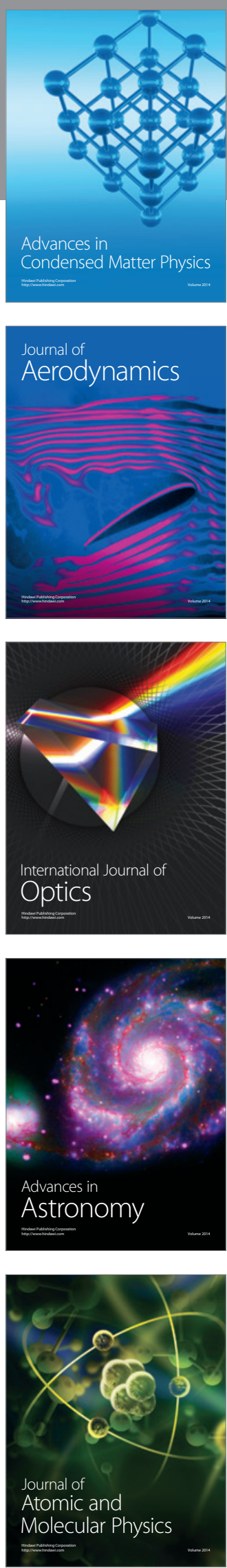